Die drängende Frage ist nicht, ob im Jahr 2050 Roboter die Weltherrschaft übernehmen und uns Menschen in Sachen Intelligenz überholen werden, oder ob man über algorithmische Formeln, die leistungsfähiger als unsere Gehirne sind, nur noch zwei Knöpfe zu unserer Unterwerfung drücken muss. Aktuell ist es tatsächlich viel wichtiger zu erkennen, dass Roboter schon jetzt Einfluss auf unser Kaufverhalten und unsere politische Überzeugung nehmen. Die Kernursache liegt aber nicht bei den autonomen Entscheidungen der Maschinen an sich, sondern an den willkürlichen Programmierungen, hinter denen echte Menschen stehen. Wenn wir wieder den Sinn der Staatsbürgerschaft erkennen wollen, dann müssen wir das zentrale Problem wieder betrachten, ohne die biotechnologischen Dilemmata $\mathrm{zu}$ ignorieren: Aus welchem Grund konzentriert sich die Kontrolle über diese Machtzusammenhänge in den Händen einiger weniger Regierungen und Unternehmen?

Die Frage, wofür sich die Algorithmen interessieren, ist unzureichend, wenn wir nicht darüber diskutieren, warum ein so kleiner Kreis an Menschen für ihre Programmierung und die Verwertung der Daten verantwortlich ist. Dieser Gedankengang zeigt uns schließlich, dass der Aufstieg der Algorithmen nicht zur Beseitigung der Ungleichheit und ihren Begleiterscheinungen führt.

\title{
Jenseits der Datenmonopolisierung
}

Wenn wir ehrlich sind, ignoriert Harari die Ungleichheit beim Zugang auf digitale Ressourcen nicht. Dem Thema »Gleichheit. Wem die Daten gehören, dem gehört die Zukunft. « widmet er ein ganzes Kapitel. Allerdings ist es, zusammen mit »Gemeinschaft«, »Gott« und »Gerechtigkeit«, mit jeweils sieben bis acht Seiten eins der kürzesten im Buch.

Er ist besorgt über die Ungleichheit, zögert aber bei der sozioökonomischen Frage: Stattdessen hält er sich an die Analyse der Datenkontrolle durch Minderheiten und überlegt, wie dadurch eine Spaltung der Gesellschaften in biologische Kasten oder verschiedene »Rassen « entstehen könnte. Die höchste Kaste würde sich laut seiner Annahme selbst isolieren, was ihren Handlungsspielraum noch unzugänglicher und unanfechtbarer machen würde, als in Zeiten des Kriegs und der politischen Abschottung. Ist eine Kontrolle dieser zunehmenden und irreversibel anmutenden Ungleichheit möglich? Der Historiker zeigt auf, dass das Eigentum der Daten ein entscheidender Faktor ist. Auffällig ist also, dass bei seinen Überlegungen die Bürger keine Rolle spie- 
len. Vielmehr wägt er ab, ob man die Datenkontrolle besser den Regierungen oder den Unternehmen anzuvertrauen sollte.

Leichte Zweifel kommen ihm dann doch: Er schreibt, er »würde [...] [seine] Daten lieber Mark Zuckerberg als Wladimir Putin anvertrauen (auch wenn der Skandal um Cambridge Analytica gezeigt hat, dass wir in dieser Frage möglicherweise gar keine Wahl mehr haben, da die Zuckerberg anvertrauten Daten möglicherweise doch irgendwann bei Putin landen).«(Harari 2018, 121).

Diese Alternative ändert sich angesichts zweier Ressourcen, die mehrere aktuelle Untersuchungen unterstreichen:

- Vermehrte Aufmerksamkeit auf die Ausweitung des Internets und der Netzwerke zur Vermeidung einer gänzlichen Auslöschung der Offlinezeit, in der die Nutzer leben.

- Angesichts der Verflechtung von Offline- und Onlineaktivitäten sollten wir unsere Aktivität in den Netzwerken so aufteilen, dass sie nicht zur Sucht werden, sondern dem Zweck des »Anschlusses an wertvolle Gemeinschaften« dienen und »unser soziales Gefüge stärken« können.

Diese zwei Ideen wurden bereits zuvor von diversen Autoren aufgegriffen. Sie stehen in Anführungszeichen, weil sie aus Mark Zuckerbergs Zeugenaussage zum Versprechen des Unternehmens Facebook stammen, zu einer globalen Community beizutragen. Facebook wurde damals von einem Skandal um gefährliche politische Auswirkungen - unter anderem während der USPräsidentschaftswahl mit dem Sieg Donald Trumps - erschüttert. Dabei kam ans Licht, dass Daten für manipulative Zwecke verkauft wurden. Diese ab 2017 propagierte »Rekonversion« ist ins Schwanken geraten. Sie verliert zudem immer mehr an Glaubwürdigkeit, seitdem ähnliche Verstrickungen in den Genozid an den Rohingya-Muslimen in Myanmar, sowie der Handel mit privaten Nutzerdaten zwischen Facebook, Apple, Amazon, Microsoft, Netflix, Spotify, der russischen und italienischen Regierung und weiteren, noch unklaren Akteuren enthüllt wurden. In den USA äußern sowohl Demokraten, als auch Republikaner ihre Besorgnis, aber weigern sich - genau wie in der Diskussion um Waffen und andere riskante Ressourcen - die Notwendigkeit einer Regulierung offen anzusprechen und voranzutreiben. Jonas Kron, Vizepräsident der Vermögensverwaltung Trillium Asset Management und Besitzer stolzer 53.000 Facebook-Aktien weist jedoch darauf hin, dass »der Mangel an Überwachung in der Firma Facebook aufgrund der mangelnden Unabhängig- 
keit des Aufsichtsrats ein enormes Risiko für das Unternehmen, seine Investoren und die Demokratie« ist (García Vega 2019, 8).

Zuckerberg verspricht, seine Unternehmenskultur zu ändern und sensationalistische Inhalte durch den Algorithmus nicht zu begünstigen. Er will Videoformate und Innovationen und Bündnisse fördern, die Nutzer weiterhin an seine Marke binden. Er äußert sich übereinstimmend mit Harari in der Annahme, dass der Schlüssel zur Gemeinschaft in einer Änderung des Geschäftsmodells liegt. Diese Vorstellung wirkt äußerst merkwürdig, wenn wir sie zur Veranschaulichung auf die erweiterte Idee des Kommunitarismus anwenden: Sie besagt, dass »jeder, von feministischen Aktivistinnen bis zu islamistischen Fundamentalisten mit Community Building beschäftigt ist« (Harari 2018, 128). 